an earned income of $£ 350,000$. But Mr Ted Leadbitter of the subcommittee still felt that the financial situation of the centre is questionable and does not support the staff of 181 (of whom about 125 have "some professional standing or some experience of the computing industry"). The National Computing Centre is represented on 73 committees, and is prolific in its publications. $\mathrm{Mr}$ Leadbitter said that the centre must be either doing its job defectively, or with a sense of being overburdened.

\section{FISH PATHOLOGY}

\section{New Laboratory}

THE disease which has disastrously afflicted salmon in the rivers of Great Britain and Northern Ireland during the past few years is to be the chief subject of research at a laboratory newly established in Weymouth on the coast of Dorset. So far the cause of the killer disease, ulcerative dermal necrosis, which is characterized externally by skin lesions, has defied identification. No bacteriological or fungal agent has been isolated, and the search is continuing at the new fish pathology laboratory, which will also serve as a source of advice and practical assistance for other laboratories, fish farmers, river authorities and so on with diseased fish to be investigated.

Although the laboratory at Weymouth was set up in a renovated coastguard's house about two years ago, in association with the Ministry of Agriculture's salmon and freshwater fisheries laboratory in London, it is not yet fully operational. The most important set of equipment, which is not quite ready for use, is an experimental unit consisting of four rooms in which fish can be quarantined in 350 gallon tanks of chilled water (sea or fresh) to ensure that toxins and infection cannot reach them. In these conditions it will be possible to investigate the transmission of ulcerative dermal necrosis and other diseases from one fish to another. Work with this experimental system will occupy much of the attention of the laboratory's staff of fifteen, which includes four fisheries scientists. When some useful results have been obtained, the study of the transmission of ulcerative dermal necrosis will move out to sea.

The possible effects of pollution on fish disease will be another important aspect of work at the laboratory, and other preoccupations will be fish haematology, histology, bacteriology, and mycology, as well as virology, which has received little attention outside the United States. The first requirement for virological research into ulcerative dermal necrosis is to develop cultures of salmon tissue; because this disease does not occur in the United States, very little is known about tissue culture of salmon. A great deal of preliminary effort is therefore being devoted to the develop- ment of basic techniques before investigations can be initiated and a possible viral cause of ulcerative dermal necrosis can be sought.

\section{COMPUTERS}

\section{Language Levels}

THE second of the 1971 Infotech State of the Art Lectures was held at the Café Royal, London, last week and will be repeated in Amsterdam on February 1618. The lectures are now well established in the computing world and the theme of the latest meeting was the rather open ended topic of high level languages and their development.

Although the past twenty years or so have seen very rapid advances in computing science, developments on the hardware side have far outstripped the relatively meagre improvements in software and programming languages. The fundamental stumbling block seems to have been the lack until recently of a scientific approach to a problem which is now seen to be neither trivial nor simple to solve -that of setting up and applying proper design criteria for high level languages. A broad definition of a high level language was that the level of a language is a measure of its independence of the machine itself (for example, the user of square root facility in ALGOL is not usually conscious of the multitude of processes which the machine performs in the course of the calculation). But this definition did not meet with unanimous approval.

It is also possible to subdivide high level languages themselves into special purpose and general purpose languages. The value of special purpose languages (such as those specifically designed to be suitable for simulation problems) was discussed by Mr P. J. Kiviat (College on Simulation and Gaming, Institution of Management Sciences, US). Although he drew attention to many of their latent disadvantages, such as availability on only a few computers, limited vendor support and the uncertain future of any one language, he emphasized that the total time from the start of programming to the output of the results could be as much as twenty times less than the equivalent time using FORTRAN, for example. This advantage has, however, to be offset against the faster machine execution time with a FORTRAN program. But once the programmer can competently handle a special purpose language, it becomes a good economic proposition, albeit for the solution of a fairly narrow range of problems.

Professor E. W. Dijkstra (Technical University of Eindhoven) probed more deeply into the reasons why software development and applications have improved so little compared with hardware. He made the interesting suggestion that the wrong qualities are being encouraged in programmers; they should break away from their image as puzzle-minded individuals looking for "clever" solutions to problems. In Dijkstra's opinion, many of the aptitude tests used to pick out good potential programmers were completely outdated and quite often the wrong type of programmer is selected. One objective should be to make programming easier and to make the detection of faults-one of the largest millstones around the programmer's necka much more efficient process. In the interests of significant improvements in software quality, he suggested several yardsticks for new high level languages which included the abolition of "go to" statements (which encourage excessive interrelationship between remote parts of the program); as well as encouraging "sequencing discipline", such a move would make debugging very much quicker.

The remarks by $\mathrm{Mr}$ A. D. Falkoff (IBM, Philadelphia) on the design of a general purpose programming language were a useful complement to Dijkstra's ideas about the needs of future languages. $\mathrm{He}$ considered that as well as being easy to learn and formally manipulable, they should be thought provoking and the documentation should "require neither a microscope to read it nor a truck to carry it". A rather controversial suggestion was that a programming language should play on the artistic instinct of the programmer so that he would develop an instinctive feeling for what was right in a particular situation. The obvious objections to Falkoff's proposals were made during the discussion, namely, that anything that was too thought provoking or that encouraged an aesthetic outlook might eventually be responsible for a great number of programming errors. Several speakers put forward the IBM general purpose language PL1 as an example of an effort to build up a general purpose language without sufficient regard for the work done on programming language theory. The result is a language which is still not in its final form and which one speaker unkindly described as "seven thousand afterthoughts in search of a language".

The interrelationship between machine design and programming languages was emphasized by two speakers with rather different points of view. $\mathrm{Mr} J$. W. Buckle (ICL) looked at the subject through the eyes of the manufacturers and emphasized the inevitable conflict between exploiting one machine for maximum efficiency and exploiting the portability of programs and data across a range of computers (such as the 1900 series) or to alien machines. $\mathrm{He}$ also spoke of the increasing call for mixed language installations and programs from which users hoped to get the best of several worlds; but, he concluded, "complexity and generality go hand in hand 